\title{
Mass calibration of the CODEX cluster sample using SPIDERS spectroscopy - II. The X-ray luminosity-mass relation
}

\section{Capasso, R.}

2020-05

Capasso , R, Mohr , J J , Saro , A, Biviano , A, Clerc , N, Finoguenov , A, Klein , M , Grandis , S , Collins , C , Damsted , S , Kirkpatrick , C \& Kukkola , A 2020 , ' Mass calibration of the CODEX cluster sample using SPIDERS spectroscopy - II. The X-ray luminosity-mass relation ', Monthly Notices of the Royal Astronomical Society , vol. 494 , no. 2 , pp. 2736-2746 . https://doi.org/10.1093/mnras/staa888

http://hdl.handle.net/10138/320661

https://doi.org/10.1093/mnras/staa888

cc_by_nc_sa

publishedVersion

Downloaded from Helda, University of Helsinki institutional repository.

This is an electronic reprint of the original article.

This reprint may differ from the original in pagination and typographic detail.

Please cite the original version. 


\title{
Mass calibration of the CODEX cluster sample using SPIDERS spectroscopy - II. The X-ray luminosity-mass relation
}

\author{
R. Capasso ${ }^{\odot, 1,2,3,4,5 \star ~ J . ~ J . ~ M o h r, ~}{ }^{1,2,6}$ A. Saro,,${ }^{3,4,7}$ A. Biviano, ${ }^{3}$ N. Clerc, ${ }^{6,8}$ \\ A. Finoguenov, ${ }^{6,9}$ M. Klein, ${ }^{1,6}$ S. Grandis,${ }^{1,2}$ C. Collins, ${ }^{10}$ S. Damsted, ${ }^{9}$ C. Kirkpatrick ${ }^{9}$ \\ and A. Kukkola ${ }^{9}$ \\ ${ }^{1}$ Faculty of Physics, Ludwig-Maximilians-Universität, Scheinerstr. 1, D-81679 Munich, Germany \\ ${ }^{2}$ Excellence Cluster Universe, Boltzmannstr. 2, D-85748 Garching, Germany \\ ${ }^{3}$ INAF-Osservatorio Astronomico di Trieste, via G. B. Tiepolo 11, I-34143 Trieste, Italy \\ ${ }^{4}$ IFPU - Institute for Fundamental Physics of the Universe, Via Beirut 2, I-34014 Trieste, Italy \\ ${ }^{5}$ The Oskar Klein Centre, Department of Physics, Stockholm University, Albanova University Center, SE-10691 Stockholm, Sweden \\ ${ }^{6}$ Max Planck Institute for Extraterrestrial Physics, Giessenbachstr. D-85748 Garching, Germany \\ ${ }^{7}$ Astronomy Unit, Department of Physics, University of Trieste, via Tiepolo 11, I-34131 Trieste, Italy \\ ${ }^{8}$ IRAP, Université de Toulouse, CNRS, UPS, CNES, 31400 Toulouse, France \\ ${ }^{9}$ Department of Physics, University of Helsinki, Gustaf Hällströmin katu 2a, FI-00014 Helsinki, Finland \\ ${ }^{10}$ Astrophysics Research Institute, Liverpool John Moores University, IC2, Liverpool Science Park, 146 Brownlow Hill, Liverpool, L3 5RF, UK
}

Accepted 2020 March 26. Received 2020 March 24; in original form 2019 October 10

\begin{abstract}
We perform the calibration of the X-ray luminosity-mass scaling relation on a sample of 344 CODEX clusters with $z<0.66$ using the dynamics of their member galaxies. Spectroscopic follow-up measurements have been obtained from the SPIDERS survey, leading to a sample of 6658 red member galaxies. We use the Jeans equation to calculate halo masses, assuming an NFW mass profile and analysing a broad range of anisotropy profiles. With a scaling relation of the form $L_{\mathrm{X}} \propto \mathrm{A}_{\mathrm{X}} M_{200 c}^{\mathrm{BX}} E(z)^{2}(1+z)^{\gamma_{\mathrm{X}}}$, we find best-fitting parameters $\mathrm{A}_{\mathrm{X}}=$ $0.62_{-0.06}^{+0.05}( \pm 0.06) \times 10^{44} \mathrm{erg} \mathrm{s}^{-1}, \mathrm{~B}_{\mathrm{X}}=2.35_{-0.18}^{+0.21}( \pm 0.09), \gamma_{\mathrm{X}}=-2.77_{-1.05}^{+1.06}( \pm 0.79)$, where we include systematic uncertainties in parentheses and for a pivot mass and redshift of $3 \times$ $10^{14} \mathrm{M}_{\odot}$ and 0.16 , respectively. We compare our constraints with previous results, and we combine our sample with the SPT SZE-selected cluster subsample observed with XMMNewton extending the validity of our results to a wider range of redshifts and cluster masses.
\end{abstract}

Key words: galaxies: clusters: general-galaxies: evolution-galaxies: kinematics and dynamics - large-scale structure of Universe.

\section{INTRODUCTION}

Accurate mass estimates of galaxy clusters are of fundamental importance for both cosmological and astrophysical studies. Observational knowledge of the mass distribution of the dark and baryonic matter in clusters provides insights into their formation and evolution (see e.g. Springel et al. 2001; Gao et al. 2004; Popesso et al. 2015; Pratt et al. 2019). On the other hand, number counts of galaxy clusters, sensitive to the amplitude of matter fluctuations, can provide constraints on various cosmological parameters in a way complementary to other cosmological probes (e.g. White, Efstathiou \& Frenk 1993; Haiman, Mohr \& Holder 2001; Mantz et al. 2015; Bocquet et al. 2019). Studies of the link between the

^E-mail: capasso@usm.lmu.de, raffaella.capasso@fysik.su.se observable features of haloes and the underlying matter distribution are thus essential.

An efficient use of clusters as cosmological probes requires a lowscatter mass proxy to relate theoretical predictions to observations (Lima \& Hu 2005; Allen, Evrard \& Mantz 2011). To infer the mass of a sample of galaxy clusters we have to be able to characterize a number of biases, depending on the intrinsic covariance of the cluster observables, measurement uncertainties, and selection effects (e.g. Pacaud et al. 2007; Mantz et al. 2010; de Haan et al. 2016). The combination of limited surveyed volume and source selection thresholds produce the well known Malmquist bias (Malmquist 1920), truncating the scattered distributions of sources in the space of observables. As a consequence, luminosity or flux selected samples are typically biased towards low masses where the selection is returning only a fraction of the underlying cluster sample. This effect is enhanced by the so-called Eddington bias (Eddington 1913). Because the number density of haloes is a steeply 
falling function of their mass (e.g. Tinker et al. 2008; Bocquet et al. 2016), the presence of scatter in the relationship between the selection observable (i.e. flux or luminosity) and mass will cause low-mass clusters to preferentially up-scatter, leading to a bias in the mass associated with the observable (Mortonson, Hu \& Huterer 2011). An accurate calibration of cluster scaling relations requires control over these biases.

Many different mass proxies have been used over the years, including thermal Sunyaev-Zeldovich effect (SZE) measurements (Staniszewski et al. 2009; Hasselfield et al. 2013; Planck Collaboration XVI 2014), weak gravitational lensing features (Corless \& King 2009; Becker \& Kravtsov 2011; Dietrich et al. 2018), cluster velocity dispersions (Biviano \& Salucci 2006; Saro et al. 2013; Capasso et al. 2019b), X-ray luminosity and temperature (Vikhlinin et al. 2009; Mantz et al. 2010; Andreon et al. 2016), and $Y_{X}$ parameter, i.e. the product of the $\mathrm{X}$-ray temperature and gas mass (Maughan 2014; Mantz et al. 2016, 2018; Truong et al. 2018). A combination of multiple, independent mass proxies help mitigate systematic uncertainties (Bocquet et al. 2015; Baxter et al. 2018; Bocquet et al. 2019; Farahi et al. 2018; McClintock et al. 2019). In a companion paper (Capasso et al. 2019a, hereafter C19) we performed the dynamical mass calibration exploiting the optical richness of a sample of 428 CODEX (COnstrain Dark Energy with X-ray clusters; Finoguenov et al. 2019) clusters, constraining the amplitude of the $\lambda$-mass relation with a $\sim 12$ per cent accuracy.

Following C19, we calibrate the X-ray luminosity-mass-redshift scaling relation by exploiting the information residing in the observed projected phase space (distribution in line of sight velocities and projected radius) of the cluster member galaxies. We use a modification of the MAMPOSSt technique (Modelling Anisotropy and Mass Profiles of Observed Spherical Systems; Mamon, Biviano \& Boué 2013), based on the Jeans equation (Binney \& Tremaine 1987), to simultaneously determine the dynamical cluster masses and the parameters of the scaling relation. The MAMPOSSt code has been successfully used to investigate the internal dynamics of clusters, determining their masses and velocity anisotropy profiles (e.g. Biviano et al. 2013, 2017; Munari, Biviano \& Mamon 2014; Capasso et al. 2019b).

We perform this analysis on the CODEX cluster catalogue, which consists of ROSAT All-Sky Survey (RASS) X-ray cluster candidates having optical counterparts in SDSS imaging data identified using the RedMaPPer algorithm (the red-sequence Matchedfilter Probabilistic Percolation algorithm; Rykoff et al. 2014). A subset of this sample has been spectroscopically studied within the SPectroscopic IDentification of eRosita Sources (SPIDERS) survey (Clerc et al. 2016). The analysis we carry out focuses on a sample of 344 CODEX clusters with a corresponding sample of $\sim 6600$ red member galaxies with measured redshifts. The clusters span the redshift range $0.03 \leq z_{\mathrm{c}} \leq 0.66$, with richnesses $20 \leq \lambda \leq 230$ and rest-frame [0.1-2.4] keV luminosities $4.5 \times 10^{42} \leq L_{\mathrm{X}} /\left(\mathrm{erg} \mathrm{s}^{-1}\right) \leq$ $3.2 \times 10^{45}$

The paper is organized as follows. In Section 2 we detail the theoretical framework. In Section 3 we present the data set used in this analysis and the selection criteria. The likelihood model used to constrain the $L_{\mathrm{X}}$-mass-redshift scaling relation is described in Section 4 , followed by the outcome of our calibration, and a discussion of a range of systematic uncertainties. We present our conclusions in Section 5.

Throughout this paper we assume a flat $\Lambda \mathrm{CDM}$ cosmology with a Hubble constant $H_{0}=70 \mathrm{~km} \mathrm{~s}^{-1} \mathrm{Mpc}^{-1}$, and a matter density parameter $\Omega_{\mathrm{M}}=0.3$. Cluster masses $\left(M_{200 \mathrm{c}}\right)$ are defined within $r_{200 c}$, the radius of the sphere inside which the cluster overdensity is
200 times the critical density of the Universe at the cluster redshift. We refer to $r_{200 c}$ as the virial radius. All quoted uncertainties are equivalent to Gaussian $1 \sigma$ confidence regions, unless otherwise stated.

\section{THEORETICAL FRAMEWORK}

We use dynamical constraints on a large ensemble of clusters to constrain the underlying halo masses, thereby enabling measurement of the luminosity-mass-redshift relation. To do this, we perform a dynamical analysis based on the application of the Jeans equation to spherical systems (Binney \& Tremaine 1987). The Jeans equation allows us to define the mass distribution $M(r)$ of a cluster as

$$
\frac{G M(<r)}{r}=-\sigma_{r}^{2}\left(\frac{\mathrm{d} \ln v}{\mathrm{~d} \ln r}+\frac{\mathrm{d} \ln \sigma_{r}^{2}}{\mathrm{~d} \ln r}+2 \beta\right),
$$

with $v(r)$ being the number density profile of the tracer galaxy population, $\sigma_{r}(r)$ the radially dependent component of the velocity dispersion along the spherical coordinate $r, M(<r)$ the enclosed mass within radius $r, G$ Newton's constant, $\beta(r) \equiv 1-\left(\sigma_{\theta}^{2} / \sigma_{r}^{2}\right)$ the radially dependent velocity dispersion anisotropy, and $\sigma_{\theta}$, one of the two (assumed identical) tangential components of the velocity dispersion.

Equation (1) can thus be used to estimate the mass distribution of a spherical system. However, the only observables we can directly obtain are projected quantities: the surface density profile of the galaxy distribution, the rest-frame LOS velocities, and the radial separation of each galaxy from the cluster centre. Because of projection effects, the determination of the mass distribution of a galaxy cluster is degenerate with the determination of the velocity anisotropy profile (e.g. Merritt 1987).

In this work, we address this problem by applying the Modelling Anisotropy and Mass Profiles of Observed Spherical Systems algorithm (hereafter MAMPOSSt; for full details please refer to Mamon et al. 2013). This method consists in determining the mass and anisotropy profiles of a cluster in parametrized form by performing a likelihood exploration of the distribution of the cluster galaxies in projected phase space, comparing it to the theoretical distribution predicted from the Jeans equation for these models. This method thus requires adopting parametrized models for the number density, mass, and velocity anisotropy profiles $v(r), M(r), \beta(r)$.

As addressed in Section 3.6, because our spectroscopic data set is likely to suffer from radially dependent incompleteness, we adopt the number density profile derived from a study of red sequence galaxies in SZE selected clusters (Hennig et al. 2017).

Regarding our choice of the mass and velocity anisotropy profiles, we follow our previous work C19. We refer to that study for a more detailed description. In the next section we summarize the main features.

\subsection{Mass and anisotropy profiles}

Driven by both numerical studies of structure formation and observational results, we adopt the mass model introduced by Navarro, Frenk \& White (1996, NFW), which is fully described by two parameters: the virial radius $r_{200}$, and the scale radius $r_{\mathrm{s}}$, which is the radius at which the logarithmic slope of the density profile is -2 . Numerous observational studies have indeed found the mass distributions of clusters to be well described by this model (Carlberg et al. 1997; van der Marel et al. 2000; Biviano \& Girardi 2003; Katgert, Biviano \& Mazure 2004; Umetsu et al. 2011). 
On the other hand, due to the lack of published studies providing strong predictions for the radial form of the velocity anisotropy profile $\beta(r)$, we consider five models that have been used in previous MAMPOSSt analyses, described also in Capasso et al. (2019b): (1) constant anisotropy model (C), (2) Tiret anisotropy profile (Tiret et al. 2007, T), (3) Mamon \& Łokas (2005) profile (MŁ), (4) Osipkov-Merritt anisotropy profile (Osipkov 1979; Merritt 1985, OM), and (5) a model with anisotropy of opposite sign at the centre and at large radii $(\mathrm{O})$.

Therefore, given a mass for each cluster we predict the projected phase space distribution of the observed dynamical data set by running MAMPOSSt with three free parameters: the virial radius $r_{200 \mathrm{c}}$, the scale radius $r_{\mathrm{s}}$ of the mass distribution, and a velocity anisotropy parameter $\theta_{\beta}$. The latter represents the usual $\beta=1-$ $\left(\sigma_{\theta}^{2} / \sigma_{r}^{2}\right)$ for the first three models $(\mathrm{C}, \mathrm{T}, \mathrm{O})$, while for the MŁ and OM models it defines a characteristic radius $\theta_{\beta}=r_{\beta}$.

\subsection{Bayesian model averaging}

As described above, we employ five velocity anisotropy models when estimating the projected phase space distribution of member galaxies for each cluster. Because we cannot strongly reject any of the models, we combine the results obtained from each anisotropy model $\beta(r)$ by merging their constraints, exploiting the Bayesian model averaging technique (see C18, Capasso et al. 2019b, for more details). In a nutshell, this method consists in assigning a weight to each model, according to how well the model fits the data. This weight is represented by the so-called Bayes factor (see Hoeting et al. 1999, and references therein).

Considering the five anisotropy models $M_{1}, \ldots, M_{5}$, the Bayes factor $B_{j}$ of each model $j$ is defined as the marginalized likelihood of the model $\mathcal{L}\left(D \mid M_{j}\right)$, also known as evidence, normalized by the likelihood of the most probable model. Specifically,

$B_{j}=\frac{\mathcal{L}\left(D \mid M_{j}\right)}{\mathcal{L}\left(D \mid M_{\max }\right)}$,

where $M_{\max }$ indicates the model with the highest marginalized likelihood, $\mathcal{L}\left(D \mid M_{j}\right)=\int \mathcal{L}\left(D \mid \theta_{j}, M_{j}\right) P\left(\theta_{j} \mid M_{j}\right) d \theta_{j}, \mathcal{L}\left(D \mid \theta_{j}, M_{j}\right)$ is the likelihood of the data $D$ given the model parameters $\theta_{j}$, and $P\left(\theta_{j} \mid M_{j}\right)$ is the prior.

The average posterior distribution of the fitted scaling relation parameters is then given by the weighted average of the posterior distributions of each model, with the Bayes factor as weight. This Bayesian model averaging is performed by means of the multimodal nested sampling algorithm MultiNest (Feroz \& Hobson 2008; Feroz, Hobson \& Bridges 2009; Feroz et al. 2019), providing us with the evidence for each model.

\section{DATA}

We perform our analysis on a subset of CODEX galaxy clusters observed within the SPIDERS survey (Clerc et al. 2016), which provides us with the spectroscopic galaxy sample. The CODEX sample is based on ROSAT All-Sky Survey (RASS, see Voges et al. 1999) selected clusters, cross-matched with nearby optically selected systems identified using the redMaPPer (the red-sequence Matched-filter Probabilistic Percolation, Rykoff et al. 2014) algorithm applied to the Sloan Digital Sky Survey IV (SDSS-IV, see Dawson et al. 2016; Blanton et al. 2017) optical imaging data. A full description of the data set construction and features are described in $\mathrm{C} 19$. In the following section we summarize the main elements of the data set.

\subsection{The CODEX sample}

The CODEX cluster sample combines ROSAT X-ray cluster candidates with optical selected cluster candidates identified using redMaPPer. First of all, RASS data are searched for all X-ray sources with detection significance $\mathrm{S} / \mathrm{N}>4$. Then, redMaPPer is run on the SDSS imaging data around each of these sources. RedMaPPer is an optical cluster-finding algorithm based on the red sequence technique, built around the richness estimator of Rykoff et al. (2012). This step thus allows the identification of candidate clusters with a red-sequence, constituting a collection of passive galaxies at a common redshift. The redMaPPer algorithm provides an estimate for the cluster photometric redshift, an estimation of the optical richness and an optical cluster centre. In cases of multiple optical counterparts meeting these criteria, the counterpart having the highest richness is assigned to the RASS X-ray source. The updated optical cluster position allows the identification of a revised red-sequence, providing the final estimate of the cluster photometric redshift and richness. Finally, RASS count rates provide an estimate for the X-ray properties of the clusters. Assuming a model for the $\mathrm{X}$-ray spectral emissivity, imposing a minimal S/N threshold of 1.6 to have optimized apertures, we calculate the aperture-corrected cluster flux $f_{X}$. From the flux, we iteratively recover the $\mathrm{X}$-ray luminosities $L_{X}$ in the rest-frame $0.1-2.4 \mathrm{keV}$ band. Starting from an initial luminosity given by the flux, we obtain an initial guess on the cluster mass and temperature using the XXL $M-T$ (Lieu et al. 2016) and $L_{X}-T$ (Giles et al. 2016) scaling relations. From the cluster mass we compute $R_{500}$ using the concentration-mass relation of Dutton \& Macciò (2014), which leads to an updated flux extraction aperture and a new estimate of the luminosity. The iteration continues until convergence is reached. For a full description of this procedure, we refer the reader to Finoguenov et al. (2019).

The final CODEX sample is then characterized by X-ray detected clusters, with estimated redshift, optical richness, optical cluster centre, and X-ray luminosity. Follow-up observations obtained with the SPIDERS survey, described below, finally provide us with spectroscopic redshift redshift measurements of cluster member galaxies.

\subsection{The SPIDERS spectroscopic sample}

The SPIDERS survey is designed to obtain homogeneous and complete spectroscopic follow-up of X-ray extragalactic sources lying within the SDSS-IV imaging footprint, with the aim of confirming galaxy cluster candidates and of assigning a precise redshift measurement. In particular, this survey was conceived to obtain follow-up observations of X-ray extended sources extracted from the all-sky X-ray eROSITA survey (extended ROentgen Survey with an Imaging Telescope Array; Predehl et al. 2010; Merloni et al. 2012). However, prior to the launch of eROSITA, the bulk of the SPIDERS program galaxy clusters is made of those identified in the shallower RASS and sparser XMM-Newton data. At the time this paper is being written, the observations of these clusters have already been completed. No further galaxy spectroscopic redshifts will be assigned to them during the final stages of the SDSS-IV program.

The target selection is performed so as to optimize the number of spectroscopically confirmed clusters. As a first step, the redMaPPer membership probability is used to assign priorities to potential targets, ranking galaxies within each cluster. The pool of targets along with the priority flag is then submitted to the eBOSS tiling 
algorithm. The eBOSS spectroscopic pipeline is then employed to produce the final data reduction and spectral classification.

For each cluster, an automatic procedure assigns membership of red-sequence galaxies with measured redshifts. This is performed through an iterative clipping procedure. Members with rest-frame velocities (relative to the first guess cluster redshift) greater than $5000 \mathrm{~km} \mathrm{~s}^{-1}$ are rejected. The remaining potential members are used to estimate the velocity dispersion of the cluster. A $3 \sigma$ clipping is then applied, rejecting objects lying further away than three times the velocity dispersion from the mean velocity.

In the course of this iterative procedure, a few problematic cases typically occur. For example, fewer than three members are sometimes assigned to a cluster, and sometimes the initial $5000 \mathrm{~km} \mathrm{~s}^{-1}$ clipping rejects all members. In such cases the problematic cluster is flagged and visually inspected by independent inspectors. This final validation may lead to the inclusion or removal of members, as well as the identification of other structures lying along line-ofsight of the cluster. Final cluster redshift estimates are based on the bi-weight average (Beers, Flynn \& Gebhardt 1990) of all galaxies selected as cluster members, if at least three members are assigned to the cluster. The cluster redshift statistical uncertainty is typically $\Delta_{z} /(1+z) \lesssim 10^{-3}$.

Finally, the updated cluster spectroscopic redshifts are used to update the measurement of X-ray cluster properties. Assuming the standard flat $\Lambda \mathrm{CDM}$ cosmological model (Hubble constant $H_{0}=70 \mathrm{~km} \mathrm{~s}^{-1} \mathrm{Mpc}^{-1}$, and matter density parameter $\Omega_{\mathrm{M}}=0.3$, ROSAT fluxes are converted into rest-frame [0.1-2.4] keV luminosities. The typical measurement uncertainty on the luminosities is $\approx 35$ percent, as computed from the Poissonian fluctuation in the associated ROSAT X-ray photons (see Mirkazemi et al. 2015).

\subsection{Final spectroscopic cluster member sample}

Before proceeding with our analysis, we apply some additional cuts to the SPIDERS spectroscopic sample. First of all, to avoid systems that are clearly in a merging stage, we only use clusters which do not have any other component along the line of sight. As we are carrying out a Jeans analysis, based on the assumption of dynamical equilibrium, we restrict our analysis to the cluster virial region $(R$ $\leq r_{200 \mathrm{c}}$ ). Moreover, we exclude the very central cluster region $(R \leq$ $50 \mathrm{kpc}$ ), to avoid the inclusion of the central BCG, in which merger and dissipation processes could be ongoing, and to account for the positional uncertainties of cluster centres. Our final spectroscopic data set consists of 705 galaxy clusters, for a total of $\approx 11400$ candidate cluster members, with a median redshift $z=0.21$ and spanning an X-ray luminosity range $4.5 \times 10^{42} \leq L_{\mathrm{X}} /\left(\mathrm{erg} \mathrm{s}^{-1}\right) \leq$ $3.2 \times 10^{45}$, and a richness range $20 \leq \lambda \leq 230$.

We take the SPIDERS validated redshifts and redMaPPer positions to calculate the observable needed for our analysis: the galaxy projected cluster-centric distance $R$ and the rest-frame line of sight (LOS) velocity $\mathrm{v}_{\mathrm{rf}}$. Rest-frame velocities are then obtained as $\mathrm{v}_{\mathrm{rf}} \equiv$ $c\left(z_{\mathrm{gal}}-z_{\mathrm{c}}\right) /\left(1+z_{\mathrm{c}}\right)$.

\subsection{Interloper rejection}

As described in Section 3.2, the SPIDERS automated procedure assesses membership for each galaxy in each cluster and subsequent visual inspection refines membership manually. However, interloper galaxies could still be present. These are galaxies that in projection are inside the cluster virial region, but do not actually lie inside it. We identify these objects by means of the 'Clean' method (Mamon

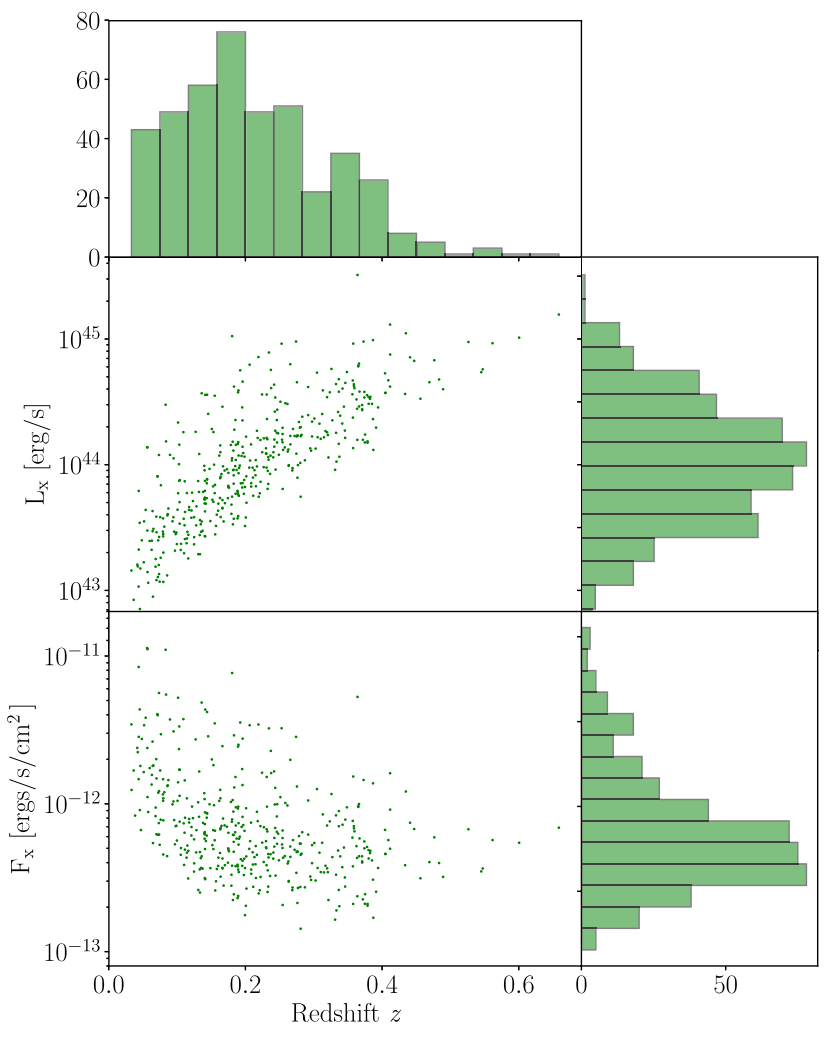

Figure 1. Distribution of X-ray luminosity $L_{X}$, flux $F_{X}$, and cluster redshift $z_{\mathrm{c}}$ of the final cluster sample.

et al. 2013), based on the comparison between the location of the galaxies in the projected phase space and the expected maximal line of sight velocity at each projected radius. As we do not have enough spectroscopic redshifts to perform this method accurately for each individual cluster, we divide our sample into 15 equally spaced $\lambda$ bins, building a composite cluster in each bin. The composite clusters are built by stacking in metric radius [Mpc], without applying any scaling in velocity. We then perform the interloper rejection in each of them separately.

The cleaning is performed in several steps. For each composite cluster, the LOS velocity dispersion $\sigma_{\mathrm{LOS}}$ is used to estimate the cluster mass $M(r)$, using a scaling relation calibrated using numerical simulations (e.g. Saro et al. 2013), and assuming an NFW mass profile with concentration sampled from the massconcentration relation. Then, assuming the MŁ velocity anisotropy profile model, and given the cluster $M(r)$, an LOS velocity dispersion profile $\sigma_{\mathrm{LOS}}(R)$ is calculated. Finally, galaxies with $\left|\mathrm{v}_{\mathrm{rf}}\right|>2.7 \sigma_{\mathrm{LOS}}$ at any cluster-centric distance are iteratively rejected (see Mamon, Biviano \& Murante 2010; Mamon et al. 2013).

After the removal of interlopers, our spectroscopic sample consists of 703 clusters and 9121 red galaxies. We apply a further cut on this data set: we only keep systems that have at least 10 spectroscopic members, $N_{\text {mem }} \geq 10$. This decision is driven by our concern that good constraints on the cluster masses and scaling relation parameters could not be obtained from clusters having very small numbers of spectroscopic members. We explore the impact of this cut in Section 4.2.3. After this cut, we are left with 428 clusters and 7807 red galaxies, with a median redshift, richness, and luminosity of $z=0.16, \lambda=41$, and $\mathrm{L}_{\mathrm{X}}=9.2 \times 10^{43} \mathrm{erg} \mathrm{s}^{-1}$, respectively. Fig. 1 shows the distributions of cluster redshift, X-ray luminosity, and flux of the final sample. 
We note that, even after this cleaning procedure, there is still a degree of contamination by interlopers. In general, galaxies lying outside the virial radius tend to have smaller peculiar velocities than those inside $R_{200}$. Galaxies close to the cluster turnaround radius will have negligible peculiar velocities, and will not be identified as interlopers by the method adopted here. An analysis of cosmological $N$-body simulations carried out by Saro et al. (2013) shows that, when passive galaxies are selected, this contamination is characteristically $\sim 20$ percent for massive clusters $\left(M_{200 \mathrm{c}} \geq\right.$ $10^{14} \mathrm{M}_{\odot}$ ), increasing with decreasing cluster mass. Another analysis carried out by Mamon et al. (2010) on hydrodynamical cosmological simulations shows that the distribution of interlopers in projected phase space is nearly universal, presenting only small trends with cluster mass. They find that, even after applying the iterative $2.7 \sigma_{\mathrm{LOS}}$ velocity cut, the fraction of interlopers is still $23 \pm 1$ percent of all DM particles with projected radii within the virial radius, and over 60 percent between 0.8 and 1 virial radius.

\subsection{Removing CODEX catalogue contamination}

When cross-matching X-ray selected candidates from RASS with optical systems from redMaPPer or other similar techniques, one must be careful to account for the contamination of the resulting cluster catalogue by random superpositions of physically unassociated X-ray and optical systems along the line of sight (Klein et al. 2018). For the RASS imaging, where there is generally no extent information for the faint CODEX sources, the contamination is driven by random superpositions between the faint X-ray sources ( $~ 90$ per cent are AGN or stars) and the ubiquitous red sequence optical candidate clusters identified by redMaPPer.

To exclude chance superpositions, we employ the method described in Klein et al. (2019). This decontamination method consists of evaluating, for a cluster candidate at redshift $z$ and richness $\lambda$, the probability distribution of richness at that redshift for detected $\mathrm{X}$-ray sources and that along random line of sights. Namely, we use the estimator $f_{\text {cont }}$, which is defined as the ratio of the integral over the two distributions, above the observed $\lambda$ of the candidate (see fig. 6 and equation 10 in Klein et al. 2019). In particular, for a given richness and redshift, we adopt the value of $f_{\text {cont, } \mathrm{m}}$ from Klein et al. (2019), which uses the distribution of observed richness together with the weighted mean of random richness distributions. We take into account differences in richness between the two RASSbased X-ray catalogues, assuming they have consistent selection and contamination properties.

We perform a cut at $f_{\text {cont, } \mathrm{m}}<0.05$, producing a sample with a 5 per cent contamination fraction, which is independent of redshift. After the cut, our final sample consists of 344 galaxy clusters with a total of 6658 cluster members, characterized by a median redshift $z=0.16$ and a median X-ray luminosity $L_{\mathrm{X}}=9 \times 10^{43} \mathrm{erg} \mathrm{s}^{-1}$. Fig. 2 shows the distribution of our sample as a function of richness and X-ray luminosity, highlighting in black clusters identified as contaminated. We exclude these objects from our analysis. In Section 4.2.1 we discuss the possible implications of this selection on our results.

\subsection{Galaxy number density profile}

As showed in Section 2, the Jeans analysis requires knowledge of the $3 \mathrm{D}$ number density profile $v(r)$ of the tracer population, i.e. the red sequence member galaxies. The absolute normalization of

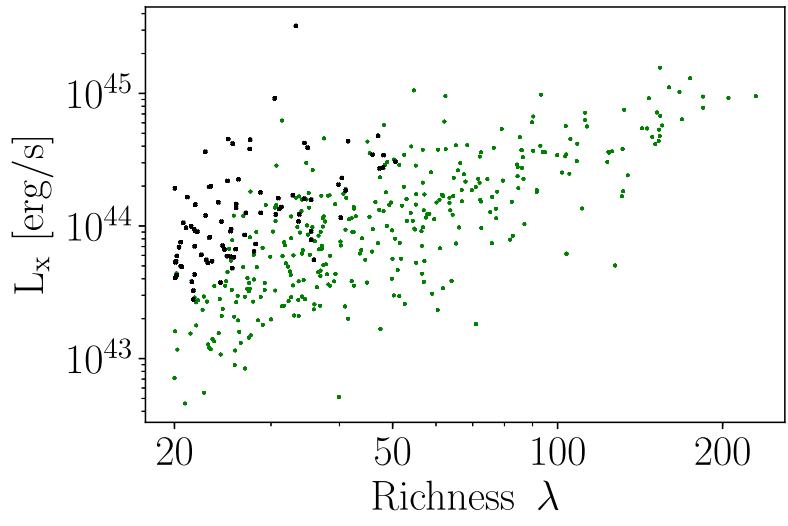

Figure 2. Distribution of X-ray luminosity and richness for our cluster sample. The black crosses represent contaminated sources, which we exclude from our analysis. We imposed the cut $f_{\text {cont, } \mathrm{m}}<0.05$, resulting in a catalogue with a 5 per cent contamination fraction.

the galaxy number density profile has no impact on our analysis, because only the logarithmic derivative of $v(r)$ enters the Jeans equation (see equation 1). On the other hand, a radially dependent incompleteness in the velocity sample would lead to a modification of the shape of the $v(r)$ profile, which would have an impact on our results. As the spectroscopic followup within SPIDERS will lead to a radially dependent incompleteness, we cannot simply adopt the spectroscopic sample to measure the number density profile of the tracer population. We therefore rely on a study of the galaxy populations in 74 SZE selected clusters from the SPT-SZ survey, imaged as part of the Dark Energy Survey Science Verification phase (Hennig et al. 2017). This study shows that the number density profile of the red sequence population is well fit by a Navarro, Frenk and White (NFW) model (Navarro et al. 1996) out to radii of $4 r_{200 \mathrm{c}}$, with a concentration for cluster galaxies of $c_{\text {gal }}=5.37_{-0.24}^{+0.27}$. No statistically significant redshift or mass trends were identified in the radial distribution of red sequence galaxies for $z>0.25$ and $M_{200 c}>4 \times 10^{14} \mathrm{M}_{\odot}$. Therefore, we adopt the number density profile described by an NFW profile with the above-mentioned value of $c_{\text {gal }}$ and a scale radius $r_{\mathrm{s} \text {, gal }}=R_{200 \mathrm{c}} / c_{\mathrm{gal}}$. Implicit in this approach is the assumption that the dynamical properties of our spectroscopic sample are consistent with those of the red sequence galaxy population analysed by Hennig et al. (2017).

We examine the impact of this assumption on our results by performing our analysis on a range of concentrations, spanning the range $3.5<c_{\text {gal }}<6$. In Appendix $\mathrm{A}$ we show that our results are not very sensitive to the choice of the concentration parameter. We will nevertheless further examine the impact of mismatch between the model and actual radial distribution of the tracer population in an upcoming study, in which we seek to improve the understanding of biases and scatter in dynamical mass estimators using mock observations of structure formation simulations (Capasso et al., in preparation).

\section{RESULTS}

This section is dedicated to the results of the dynamical analysis. In the first subsection we present the method used to calibrate the $L_{X}$-mass relation, and the results we obtain. We end the section with a comparison of our findings to those from previous studies, and we discuss the impact of the choice of the priors on our results. 


\subsection{Fitting procedure}

We model the relation between the X-ray luminosity, mass and redshift as

$\frac{L_{\mathrm{X}}}{\left(10^{44} \mathrm{erg} \mathrm{s}^{-1}\right)}=\mathrm{A}_{\mathrm{X}}\left(\frac{M_{200 \mathrm{c}}}{M_{\text {piv }}}\right)^{\mathrm{BX}_{\mathrm{X}}}\left(\frac{E(z)}{E\left(z_{\text {piv }}\right)}\right)^{\mathrm{C}_{\mathrm{X}}}\left(\frac{1+z}{1+z_{\text {piv }}}\right)^{\gamma \mathrm{X}}$,

where $A_{X}, B_{X}$, and $\gamma_{X}$ are the amplitude, the mass slope, and the redshift evolution slope. In this formulation, the redshift trend is expressed as both a function of $z$, and of the Hubble parameter $H(z)=H_{0} E(z)$. In this analysis, we fix $\mathrm{C}_{\mathrm{X}}=2$. In a flat $\Lambda \mathrm{CDM}$ Universe, $E^{2}(z)=\Omega_{\mathrm{m}}(1+z)^{3}+\Omega_{\Lambda}$ at late times. Therefore, we explicitly parametrize the cosmological dependence of the redshift evolution, while modelling departures from the self-similar evolution with a function $(1+z)^{\gamma x}$. Similar forms have been previously adopted to study the redshift and mass trends of the $L_{\mathrm{X}}$-mass relation (e.g. Bulbul et al. 2019). The redshift and mass pivot points are set to be $z_{\text {piv }}=0.16$ and $M_{\text {piv }}=3 \times 10^{14} \mathrm{M}_{\odot}$, respectively, corresponding to the median mass and redshift of our sample, where the $M_{\text {piv }}$ has been chosen a posteriori, after a first run of the analysis. We adopt a lognormal intrinsic scatter in $L_{X}$ at fixed mass, $\sigma_{\ln L_{X}}^{\text {int }}$.

We follow the fitting framework presented in C19. Given the set of parameters $\boldsymbol{p}$, containing the four scaling relation parameters $\left(A_{\mathrm{X}}\right.$, $\left.B_{\mathrm{X}}, \gamma_{\mathrm{X}}, \sigma_{\ln \lambda}^{\text {int }}\right)$ and the anisotropy model parameter $r_{\beta}$, we calculate an initial mass $M_{200 \text { c, obs }}$ using the scaling relation presented in equation (3). We then use the method of Mortonson et al. (2011) to estimate the Eddington bias correction caused by the interplay of the cluster mass function and scatter of the scaling relation. We assume the variance on the lognormal mass-observable relation to be $\sigma_{\ln M}^{2}=\left(1 / B_{\mathrm{X}} \cdot \sigma_{\ln L_{\mathrm{X}}}\right)^{2}$, where

$\sigma_{\ln L_{X}}^{2}=\left(\frac{\Delta L_{X}}{L_{X}}\right)^{2}+\sigma_{\ln L_{X}}^{\text {int }}{ }^{2}$,

with $\Delta L_{\mathrm{X}}$ being the $L_{\mathrm{X}}$ measurement uncertainty divided by the observed luminosity. Assuming that the variance $\sigma_{\ln M}^{2}$ is small compared with the scale over which the local slope $\Gamma$ of the mass function changes, the posterior mass distribution is a lognormal of the same variance $\sigma_{\ln M}^{2}$ with the mean shifting as $\ln <M_{200 \text { c,true }}>=\ln <M_{200 \text { c,obs }}>+\Gamma \sigma_{\ln M}^{2}$. We adopt this mass as input in MAMPOSSt, evaluating for each cluster the likelihood distribution in projected phase space. We combine the likelihoods calculated for each member galaxy in that cluster, such that the $i-$ th term in the likelihood $\mathcal{L}_{i}$ contains the probability of observing the $i$

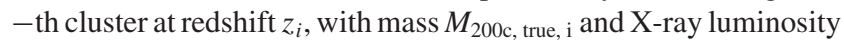
$L_{\mathrm{X}, \mathrm{i}}$, and the phase space of its member galaxies (cluster-centric radii $R^{j}$ and rest-frame velocities $v_{r f}^{j}$ of each $j$-th galaxy), given the scaling relation parameters $\mathrm{A}_{\mathrm{X}}, \mathrm{B}_{\mathrm{X}}$, and $\gamma_{\mathrm{X}}$, the anisotropy parameter $r_{\beta}$, and the intrinsic scatter $\sigma_{\ln L_{X}}^{\text {int }}$ :

$\mathcal{L}_{i}=\prod_{j \in g a l} \mathcal{L}\left(R^{j}, v_{r f}^{j}, L_{\mathrm{X}, \mathrm{i}}, z_{i} \mid \mathbf{p}\right)$.

The maximum likelihood solutions are obtained using the NEWUOA software (Powell 2006). Priors on the parameters are assumed as follows (see Table 1): flat for the scaling relation parameters and for the anisotropy parameter, Gaussian for the intrinsic scatter (mean $\mu=0.27$ and variance $\sigma^{2}=0.1^{2}$, from Bulbul et al. 2019).

The final likelihood for the total sample, for each set of scaling relation parameters $\boldsymbol{p}$, will then be obtained by combining the likelihoods for all the single clusters:

$\mathcal{L}=\prod_{i \in \text { clus }} \mathcal{L}_{i}$
Table 1. Priors assumed for our analysis. $\mathcal{U}(i, j)$ refers to a uniform flat prior in the interval $(i, j)$, while $\mathcal{N}\left(\mu, \sigma^{2}\right)$ indicates a Gaussian distribution with mean $\mu$ and variance $\sigma^{2}$.

\begin{tabular}{lcccc}
\hline $\mathrm{A}_{\mathrm{L}_{\mathrm{X}}}$ & $\mathrm{B}_{\mathrm{L}_{\mathrm{X}}}$ & $\gamma_{L_{\mathrm{X}}}$ & $\theta_{\beta}$ & $\sigma_{\ln L_{\mathrm{X}}}$ \\
\hline $\mathcal{U}(0.1,2)$ & $\mathcal{U}(1,5)$ & $\mathcal{U}(-7,2)$ & $\mathcal{U}(0.01,10)$ & $\mathcal{N}\left(0.27,0.1^{2}\right)$ \\
\hline
\end{tabular}

This procedure is carried on separately for each anisotropy profile model (see Section 2.1). The posterior parameter distributions obtained from the different anisotropy models are then combined by means of the Bayesian model averaging technique, effectively marginalizing over the uncertainties in the orbital anisotropy (see discussion in Section 2.2).

\subsection{Systematic effects}

This section is dedicated to estimating the systematic errors entering our analysis and their impact on the best-fitting parameter uncertainties.

\subsubsection{Selection bias}

As described in Section 3.1 (see also the bottom panel of Fig. 1), the sample analysed in this work is mainly flux-limited, with a further richness selection. This selection could introduce a bias into our analysis. To estimate the impact of this systematic bias on our results, we estimate its effects on a mock sample.

Starting from the halo mass function (Tinker et al. 2008), we create a large mock catalogue, computing the number of expected clusters as a function of halo mass and redshift $\left(\sim 3.6 \times 10^{6}\right.$ clusters). We draw a Poisson realization of this data set, obtaining a mass selected sample made of $\sim 800$ clusters (doubling the observed data set), with $M_{200 \mathrm{c}} \geq 5 \times 10^{13}$ and $0.05 \leq z \leq 0.66$. To each cluster we assign a luminosity sampled from a Gaussian distribution centred on the X-ray luminosity predicted by our analysis using one anisotropy model, namely the constant anisotropy model, and scatter given by $\sigma_{\ln L_{X}}^{\mathrm{int}}$. The input values for the scaling relation, which thus slightly differ from the values listed in Table 2, are as follows: $A_{\mathrm{X}}=0.54, \mathrm{~B}_{\mathrm{X}}=2.37$, and $\gamma_{\mathrm{X}}=-2.41$. To create the sample of member galaxies for each cluster, we run MAMPOSSt on a grid of velocities and radii, fixing the galaxy number density profile to that described in Section 3.6. From the likelihood we derive the probability density of observing an object at a certain projected phase space location (see equation 11, Mamon et al. 2013), drawing a random number of galaxies from the observed distribution of member galaxies. We fit this mock sample following the procedure described in Section 4.1, recovering best-fitting parameters consistent with the input values. As a second step, we convert the $\mathrm{X}$-ray luminosity in flux using the equation $L_{\mathrm{X}} /\left(4 \pi \mathrm{D}_{\mathrm{L}}{ }^{2}\right)$, where $\mathrm{D}_{\mathrm{L}}$ is the luminosity distance at fixed cosmology and redshift. Finally, we calculate richness using the scaling relation calibrated in C19. To estimate the effect of the selection bias, we impose the same cuts that are applied to the observed sample, i.e. a flux cut at $F_{\mathrm{X}}>$ $1.4 \times 10^{-13}\left[\mathrm{erg} \mathrm{s}^{-1} \mathrm{~cm}^{-2}\right]$, and a richness cut at $\lambda>20$ combined with a redshift-dependent richness cut due to the removal of random superposition (see blue line in Fig. 3). Performing our analysis on this mock sample, consisting of $\sim 800$ clusters and $\sim 22400$ member galaxies, we recover the following constraints on the scaling relation parameters: $A_{\mathrm{X}}=0.53_{-0.02}^{+0.02}, \mathrm{~B}_{\mathrm{X}}=2.45_{-0.09}^{+0.09}$, and $\gamma_{\mathrm{X}}=-2.58_{-0.58}^{+0.52}$. These values are less than $1 \sigma$ away from the input parameters, and small compared to the statistical uncertainties reported in Table 2. 
Table 2. X-ray luminosity-mass-redshift scaling relation parameters and intrinsic scatter from this analysis and the literature. Parameters are as defined in equation (3), and include the Eddington and Malmquist biases. Results from this analysis are showed with statistical uncertainties, together with systematic mass uncertainties. In the comparison to previous results, the amplitude $\mathrm{A}_{\mathrm{X}}$ column contains the luminosity at $M_{200 \mathrm{c}}=3 \times 10^{14} \mathrm{M}_{\odot}$ and $z=0.16$. Conversions have been made to $M_{200 \mathrm{c}}$ and from $E(z)$ to $(1+z)$ where needed. Note also that each of these studies was performed on a different range of mass and redshift.

\begin{tabular}{|c|c|c|c|c|c|}
\hline Dynamical analysis using SPIDERS data & $A_{X}$ & $\mathrm{~B}_{\mathrm{X}}$ & $\gamma_{\mathrm{X}}$ & $\mathrm{C}_{\mathrm{X}}$ & $\sigma_{\ln L_{X}}^{\mathrm{int}}$ \\
\hline Combined analysis & $0.60_{-0.06}^{+0.05}$ & $2.01_{-0.09}^{+0.09}$ & $-0.56_{-0.36}^{+0.36}$ & 2 & $0.23_{-0.08}^{+0.09}$ \\
\hline \multicolumn{6}{|l|}{ Previously published results } \\
\hline SPT + XMM-Newton (Bulbul et al. 2019) & $0.58 \pm 0.09$ & $1.92 \pm 0.18$ & $0.004 \pm 0.50$ & 2 & $0.27 \pm 0.10$ \\
\hline WL + RASS (Nagarajan et al. 2019) & $0.42 \pm 0.27$ & $1.62 \pm 0.30$ & - & 2 & $0.75_{-0.16}^{+0.19}$ \\
\hline Chandra + ROSAT (Vikhlinin et al. 2009) & $0.68 \pm 0.21$ & $1.63 \pm 0.15$ & - & $1.85 \pm 0.42$ & $0.40 \pm 0.04$ \\
\hline REXCESS (Pratt et al. 2009) & $1.04 \pm 0.09$ & $1.63 \pm 0.11$ & - & 2.33 & $0.41 \pm 0.07$ \\
\hline
\end{tabular}

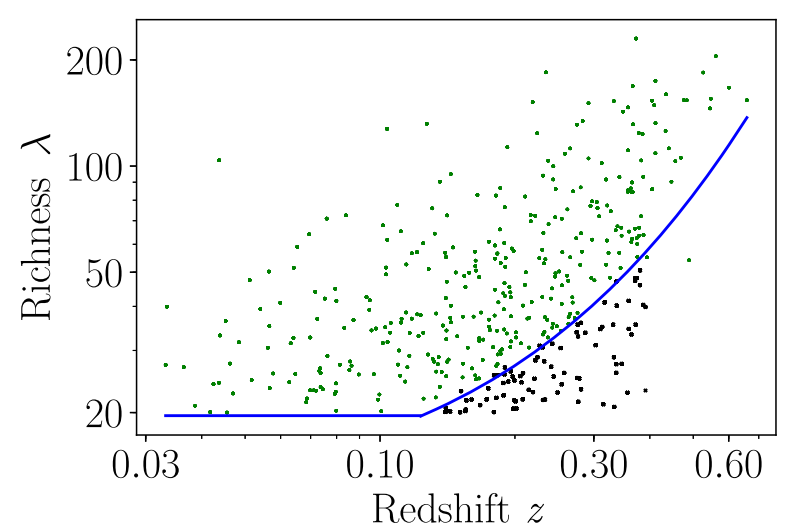

Figure 3. Distribution of our cluster sample in richness and redshift. The black crosses represent the contaminated sources that we exclude from the sample. The richness sample selection is shown by the blue line.

We conclude our results are not significantly affected by a selection bias.

\subsubsection{Systematics in MAMPOSSt mass estimates}

Another systematic effect we need to take into account is the one associated with the dynamical mass measurements themselves. To estimate this additional systematic uncertainty we employ the findings of Mamon et al. (2013), recovered by analysing runs of the MAMPOSSt code on numerical simulations. Using particles lying within a sphere of $r_{100}$ around the halo centre, they show that the estimated value of the cluster virial radius $r_{200 \mathrm{c}}$ is biased at $\leq 3.3$ per cent (see Table 2; Mamon et al. 2013). We thus adopt a Gaussian systematic uncertainty on the virial mass $M_{200 \mathrm{c}}$ of $\sigma=10$ per cent. As the Mamon et al. (2013) analysis does not explore mass or redshift trends in these biases, we apply the entire uncertainty to the normalization parameter $A_{X}$. In a future analysis, we plan to explore the mass and redshift dependence of the systematic uncertainties in dynamical mass estimates from a Jeans analysis on numerical simulations.

\subsubsection{Impact of the number of member galaxies}

As mentioned in Section 3.4, we apply a cut on the number of spectroscopic members per cluster, $N_{\text {mem }} \geq 10$. This choice derives from the concern that below a certain number of cluster members
Table 3. Impact of the number of member galaxies on the luminositymass-redshift scaling relation parameters, defined in equation (3). The uncertainties on the results are statistical, corresponding to 68 percent confidence intervals.

\begin{tabular}{lccc}
\hline $\begin{array}{l}\text { Number of cluster } \\
\text { member galaxies }\end{array}$ & $\mathrm{A}_{\mathrm{X}}$ & $\mathrm{B}_{\mathrm{X}}$ & $\gamma_{\mathrm{X}}$ \\
\hline$N_{\text {mem }} \geq 1$ & $0.64_{-0.06}^{+0.05}$ & $2.20_{-0.16}^{+0.18}$ & $-1.19_{-0.80}^{+0.73}$ \\
$N_{\text {mem }} \geq 3$ & $0.64_{-0.06}^{+0.05}$ & $2.24_{-0.17}^{+0.19}$ & $-1.39_{-0.89}^{+0.83}$ \\
$N_{\text {mem }} \geq 5$ & $0.64_{-0.06}^{+0.05}$ & $2.27_{-0.16}^{+0.17}$ & $-1.75_{-0.83}^{+0.81}$ \\
$N_{\text {mem }} \geq 10$ & $0.62_{-0.06}^{+0.05}$ & $2.35_{-0.18}^{+0.21}$ & $-2.77_{-1.05}^{+1.06}$ \\
$N_{\text {mem }} \geq 15$ & $0.59_{-0.06}^{+0.05}$ & $2.38_{-0.21}^{+0.25}$ & $-2.52_{-1.30}^{+1.14}$ \\
\hline
\end{tabular}

even the mean redshift of the cluster becomes uncertain and the dynamical information becomes too noisy to be reliable for a scaling relation reconstruction. Following C19, we estimate the impact of this cut on our results.

Table 3 lists the constraints on the best-fitting parameters for varying values of $N_{\text {mem }}$, from 1 to 15 . We note that, as the BCG has already been excluded, clusters with $N_{\text {mem }}=1$ actually have two measured spectroscopic redshifts. As in $\mathrm{C} 19$, this cut does not significantly affect the normalization $A_{X}$ and the mass trend parameter $\mathrm{B}_{\mathrm{X}}$. On the other hand, the redshift trend parameter $\gamma_{\mathrm{X}}$ changes considerably. The value of $\gamma_{X}$ starts converging when including only clusters with at least 10 spectroscopic members, justifying the cut imposed on our sample.

The strong dependence of $\gamma_{X}$ on the number of galaxies could indicate an additional source of systematic uncertainty. In the discussion presented in $\mathrm{C} 19$, we highlight that the distribution of clusters with $N_{\text {mem }}<10$ extends to higher redshifts, representing a qualitatively different population of objects. To assess whether the trend in $\gamma_{\mathrm{X}}$ represents the true redshift trend or is a sign of a systematic in the limit of low spectroscopic sampling, further exploration with a larger high $z$ spectroscopic sample is needed. In the meantime, we use this apparent trend to estimate a systematic uncertainty on the scaling relation parameters. As in C19, we define this uncertainty as half the full range of variation in the value of the parameter, $\sigma_{\mathrm{sys}, \gamma_{\mathrm{X}}}=\frac{\Delta\left|\gamma_{\mathrm{X}}\right|}{2}=0.79$. We also estimate this factor for the mass trend parameter, $\sigma_{\mathrm{sys}, \mathrm{B}_{\mathrm{X}}}=\frac{\Delta|\mathrm{BX}|}{2}=0.09$. For the amplitude parameter, on the other hand, the shift is small compared to the 10 percent systematic uncertainty described at the beginning of this section. These systematic uncertainties are included in results listed in Table 2. 


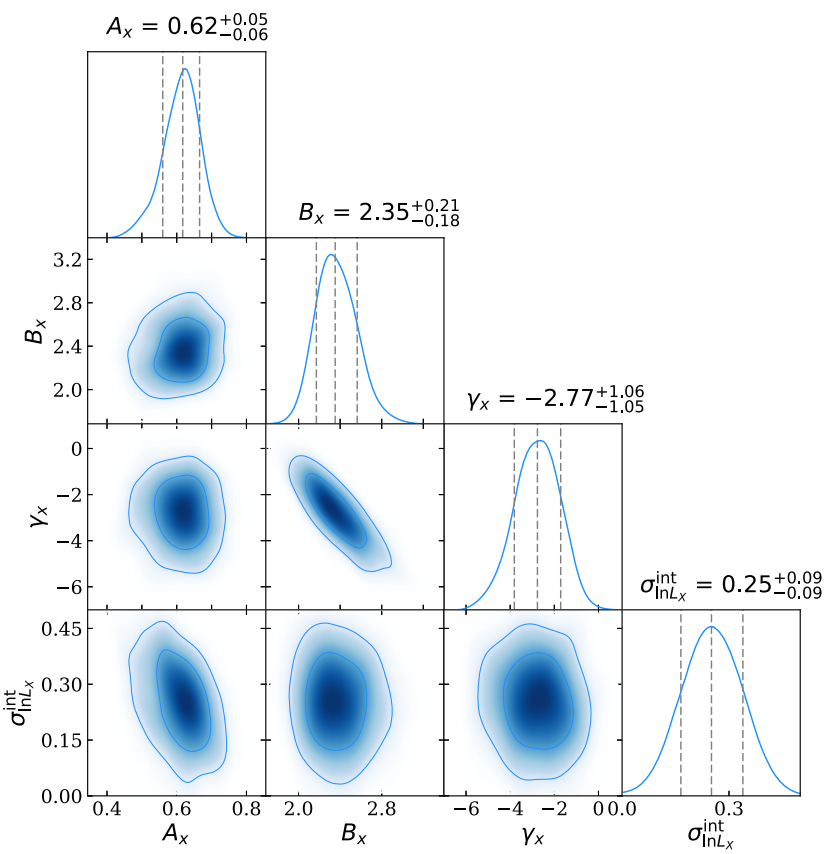

Figure 4. Posterior distribution of the scaling relation parameters. Contours show the $1 \sigma, 2 \sigma$, and $3 \sigma$ confidence regions.

\subsection{Parameter constraints}

The resulting posteriors of our scaling relation parameters are summarized in Table 2. The uncertainties are statistical, together with the additional 10 percent systematic uncertainty described above. Fig. 4 shows the corresponding joint parameter constraints. We find that galaxy clusters with mass $M_{200 \mathrm{c}}=3 \times 10^{14} \mathrm{M}_{\odot}$ at $z=0.16$ have a mean X-ray luminosity $L_{\mathrm{X}}=0.62_{-0.06}^{+0.05} \times 10^{44}$, and scale with mass and redshift as $\mathrm{B}_{\mathrm{X}}=2.35_{-0.18}^{+0.21}$ and $\gamma_{\mathrm{X}}=-2.77_{-1.05}^{+1.06}$ respectively. The posterior distribution of the intrinsic scatter is consistent with that of the prior. In the following section we compare our calibration of the $L_{X}$-mass relation to previous results from the literature.

\subsection{Comparison to previous results}

Table 2 summarizes the parameter estimates and uncertainties for our analysis and the Bulbul et al. (2019) comparison results. To make this comparison, we scale the measurements from Bulbul et al. (2019) to the redshift $z_{\text {piv }}=0.16$, and mass $M_{\text {piv }}=3 \times 10^{14} \mathrm{M}_{\odot}$, using the published best-fitting redshift and mass trends. The mass conversion from $M_{500 \mathrm{c}}$ to $M_{200 \mathrm{c}}$ is carried out using COLOSSUS, an open-source python package for calculations related to cosmology (Diemer 2018). Moreover, the analysis performed in Bulbul et al. (2019) is also based on luminosities extracted within $r_{500}$ (referred to as core-included), but in a rest-frame band of [0.5-2] keV. We thus estimate a factor 1.6 to be applied to their amplitude.

Figs 5 and 6 show the mass and redshift trends of the X-ray luminosity, where for the redshift trend we correct the data points to the mass $M_{200 \mathrm{c}}=6 \times 10^{14} \mathrm{M}_{\odot}$ and for the mass trend we move the data points to the redshift $z=0.4$. These values have been chosen as clusters with such mass at this redshift are present in both our data set and the one analysed by Bulbul et al. (2019). This means that these are not the places where our constraints are tightest, but the ones where the comparison between the works is justifiable. The best-fit model for the $L_{\mathrm{X}}-M_{200 \mathrm{c}}$ relation is shown in red, with

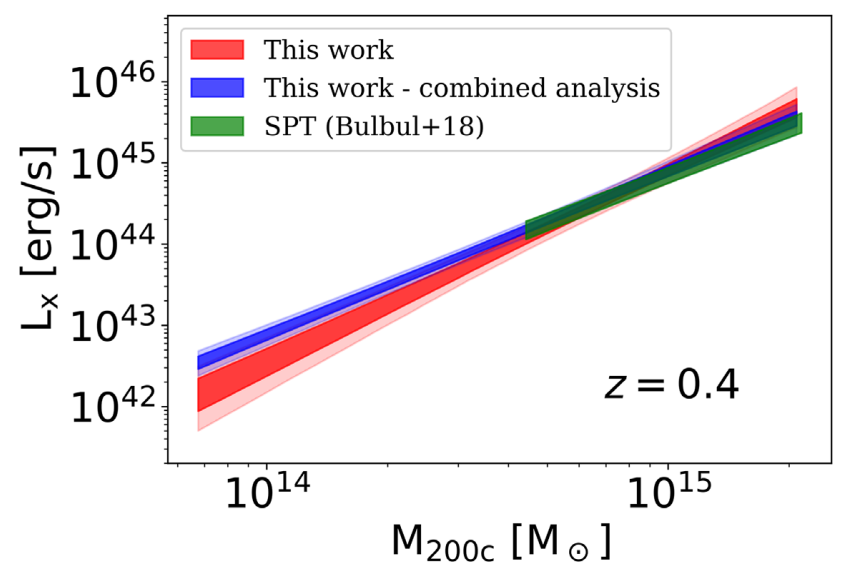

Figure 5. Best-fitting model for our X-ray luminosity-mass relation (in red), evaluated at the redshift $z=0.4$, compared to the Bulbul et al. (2019) measurements. In blue we show the results from a combined analysis of the two results. The shaded regions correspond to the $1 \sigma$ and $2 \sigma$ confidence regions. For the Bulbul et al. (2019) results we only show the $1 \sigma$ confidence area.

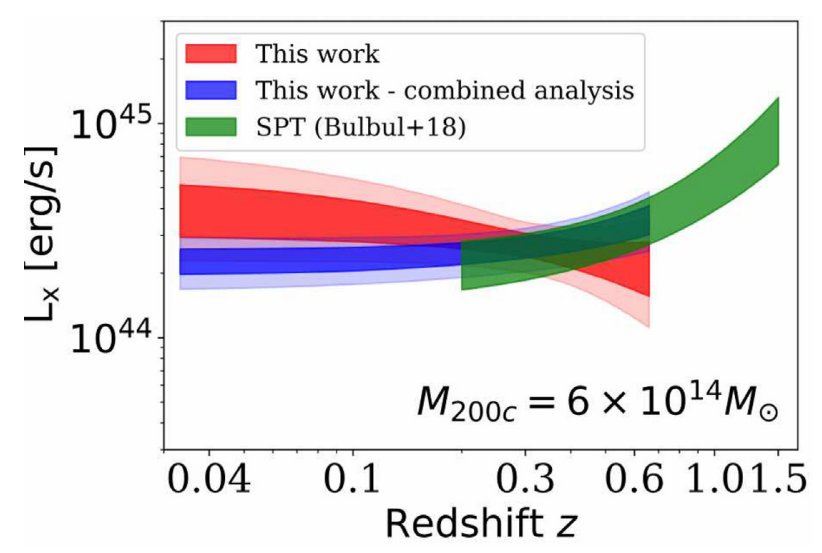

Figure 6. Best-fitting model for our X-ray luminosity-mass relation (in red), evaluated at the mass $M_{200 c}=6 \times 10^{14} \mathrm{M}_{\odot}$, compared to the Bulbul et al. (2019) measurements. In blue we show the results from a combined analysis of the two results. The shaded regions correspond to the $1 \sigma$ and $2 \sigma$ confidence regions. For the Bulbul et al. (2019) results we only show the $1 \sigma$ confidence area.

shaded $1 \sigma$ and $2 \sigma$ confidence regions. For the results from Bulbul et al. (2019, in green), we show only the $1 \sigma$ confidence region. We limit the redshift range to that analysed in each work.

Our mass trend shows good agreement with the results obtained by Bulbul et al. (2019), based on XMM-Newton X-ray observations of an SZE selected sample from the South Pole Telescope $2500 \mathrm{deg}^{2}$ SPT-SZ survey (Fig. 5). We also find a good agreement with the results reported from weak-lensing derived masses of an X-ray selected sample (APEX-SZ; Nagarajan et al. 2019). Additionally, our mass trend is consistent with that found by Giles et al. (2017), obtained through an analysis of galaxy clusters observed with Chan$d r a$, and Vikhlinin et al. (2009), based on Chandra observations of samples derived from the ROSAT All-Sky survey. However, we find a steeper $\mathrm{B}_{\mathrm{X}}$ compared to that reported in Mantz et al. (2016), also based on Chandra and ROSAT data. We also compare our mass trend with that found by Pratt et al. (2009), based on the Representative XMM-Newton Cluster Structure Survey (REXCESS) data set. We find our scaling relation to be somewhat steeper (at a $3.3 \sigma$ level) 


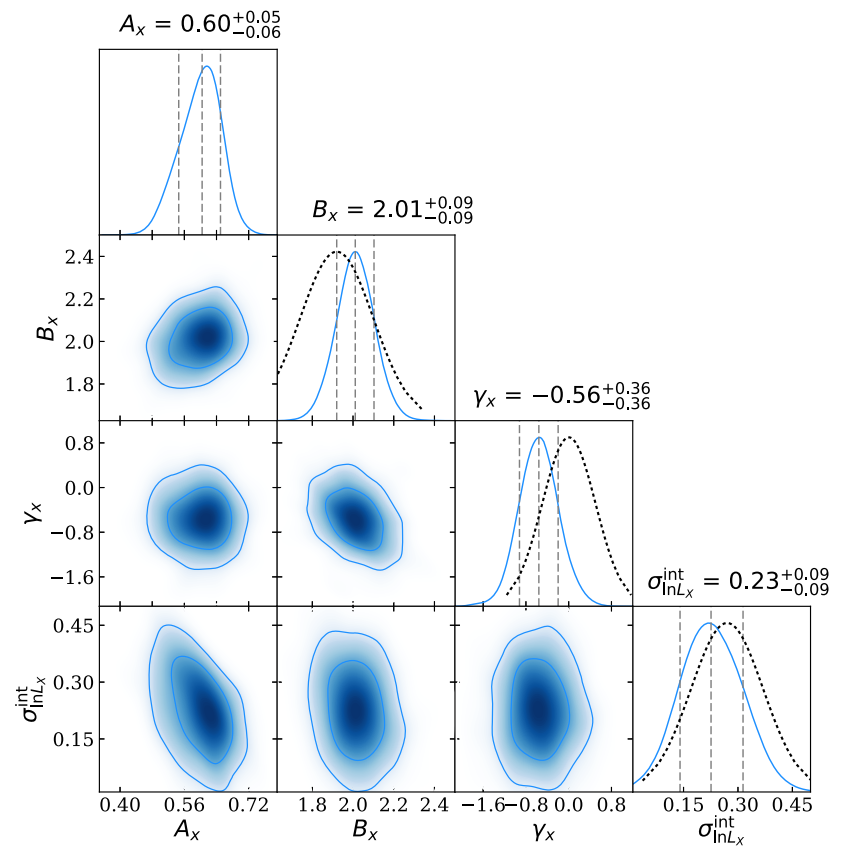

Figure 7. Posterior distribution of the scaling relation parameters for the combined analysis. Contours show the $1 \sigma, 2 \sigma$, and $3 \sigma$ confidence regions, also highlighted by the vertical dashed lines. The prior distributions for the mass and redshift trends and the intrinsic scatter are taken from Bulbul et al. (2019) and shown in black. The prior on the normalization is the flat one showed in Table 1.

than their [0.1-2.4] keV band scaling relation, corrected for the Malmquist bias. Overall, our study recovers a steeper than selfsimilar mass trend, in agreement with most previously published analyses.

Our constraint on the redshift trend of the $L_{\mathrm{X}}-M_{200 \mathrm{c}}-z$ relation, on the other hand, suggests a stronger negative evolution than found by Bulbul et al. (2019) (Fig. 6). However, we note that the redshift range probed by Bulbul et al. (2019) is higher and complementary to that covered by our sample. In Section 4.5 we describe the results obtained by combining the two samples. Our redshift trend (both from our baseline analysis and the combined one) is also in good agreement with the value of $\gamma_{X}$ found by Mantz et al. (2016). We also note that all the results from the literature we cited assume a self-similar evolution of the form $E(z)^{2}$, apart from Mantz et al. (2016), Vikhlinin et al. (2009), and Pratt et al. (2009). For a discussion of the expected self-similar trends in mass and redshift, we refer the reader to Bulbul et al. (2019).

\subsection{Combined analysis}

Our sample and the one analysed by Bulbul et al. (2019) cover complementary ranges of mass and redshift. In particular, the SPT selected cluster sample extends to higher redshift, and is therefore helpful in constraining the redshift evolution parameter of the scaling relation. Therefore, we perform a 'combined' analysis by adopting the priors on the mass and redshift trends found by Bulbul et al. (2019). Fig. 7 shows the posterior distribution of the scaling relation parameters, together with the prior distributions for the mass and redshift trends and the intrinsic scatter. We note that the two sets of distributions are in agreement, allowing us to perform this joint analysis. The prior on the normalization is the same flat prior used for the CODEX-only analysis (see Table 1). The results are listed in Table 2. Figs 5-7 all demonstrate that the results of the combined analysis are fully consistent with the Bulbul et al. (2019) ones, showing a shallower mass trend and a higher value of the redshift trend.

\section{CONCLUSIONS}

We present the calibration of the X-ray luminosity-mass-redshift relation using galaxy dynamical information from a sample of 344 CODEX galaxy clusters. These systems are X-ray selected clusters from RASS that have red-sequence selected redMaPPer optical counterparts within a search radius of $3 \mathrm{arcmin}$. The sample is cleaned of random superpositions using an $f_{\text {cont }}=0.05$ cut (Klein et al. 2019), which reduces the contamination from an initial $\sim 25$ per cent to a target 5 per cent. The cluster sample we analyse has redshifts up to $z \sim 0.66$, optical richness $\lambda \geq 20$, and spans an $X$ ray luminosity range $4.5 \times 10^{42} \leq L_{\mathrm{X}} /\left(\mathrm{erg} \mathrm{s}^{-1}\right) \leq 3.2 \times 10^{45}$. The spectroscopic follow-up has been obtained from the SPectroscopic IDentification of eRosita Sources (SPIDERS) survey, resulting in a final sample of 6658 red member galaxies.

We perform a Jeans analysis based on the code MAMPOSSt (Mamon et al. 2013). For each individual cluster, we extract the likelihood of consistency between the projected phase space distribution of the cluster members with measured redshifts and the modelled projected distribution for a cluster at redshift $z$, luminosity $L_{\mathrm{X}}$, and inferred mass $M_{200 \mathrm{c}}$. We adopt an NFW profile for the red galaxy tracer population with concentration $c=5.37$ (Hennig et al. 2017, and Section 3.6), and employ five different velocity dispersion anisotropy profiles. We combine luminosity-mass relation posterior parameter distributions from the different anisotropy models by performing Bayesian model averaging, allowing us to marginalize over the orbital anisotropy of the spectroscopic galaxy population.

The scaling relation is modelled as $L_{\mathrm{X}} \propto \mathrm{A}_{\mathrm{X}} M_{200 \mathrm{c}}^{\mathrm{B}_{\mathrm{X}}} E(z)^{2}(1+$ $z)^{\gamma \times}$ (equation 3 ). We correct for the Eddington bias by implementing the method described in Mortonson et al. (2011), which provides an estimate of the mean mass shift due to the lognormal mass observable relation scatter (equation 4) together with the measurement uncertainties on the X-ray luminosity. We also correct for the Malmquist bias, after evaluating its effect on a mock sample.

Results are showed in Table 2. For clusters of mass $M_{\text {piv }}=3 \times$ $10^{14} \mathrm{M}_{\odot}$, at redshift $z_{\text {piv }}=0.16$, we find the following constraints on the scaling relation parameters:

$$
\begin{aligned}
& \mathrm{A}_{\mathrm{X}}=0.62_{-0.06}^{+0.05} \pm 0.06, \\
& \mathrm{~B}_{\mathrm{X}}=2.35_{-0.18}^{+0.21} \pm 0.09, \\
& \gamma_{\mathrm{X}}=-2.77_{-1.05}^{+1.06} \pm 0.79,
\end{aligned}
$$

where we quote systematic uncertainties for all the parameters. The amplitude uncertainty of 10 percent comes from an estimate of the dynamical mass systematic uncertainty, applied to the scaling relation amplitude $\mathrm{A}_{\mathrm{X}}$ (see study of systematics in Mamon et al. 2013).

Our results on the mass trend of the scaling relation are steeper, but statistically consistent (within $3 \sigma$ ) with some previous literature results (Vikhlinin et al. 2009; Giles et al. 2017; Nagarajan et al. 2019; Bulbul et al. 2019). However, we find mild disagreement with results from Pratt et al. (2009) (at 3.3 $\sigma$ level) and large departures from the Mantz et al. (2016) mass trend.

We examine the redshift trend of the $L_{X}$-mass scaling relation, finding a stronger negative, non-self-similar evolution of $L_{X}$ with redshift with respect to the Bulbul et al. (2019) results. We explore this result by performing our analysis fixing the priors on our mass 
and redshift slopes to those adopted in Bulbul et al. (2019). We recover the following set of parameters:

$$
\begin{aligned}
\mathrm{A}_{\mathrm{X}} & =0.60_{-0.06}^{+0.05}, \\
\mathrm{~B}_{\mathrm{X}} & =2.01_{-0.09}^{+0.09}, \\
\gamma_{\mathrm{X}} & =-0.56_{-0.36}^{+0.36} .
\end{aligned}
$$

We note that the redshift trend has shifted to significant higher values, being consistent with the self-similar evolution and with previous studies.

This work, together with $\mathrm{C} 19$, shows the potential of dynamical masses in deriving mass-observable relations even in the limit of a small number of cluster members. This very promising result will be extremely useful in the context of future spectroscopic surveys like DESI (Levi et al. 2013), 4MOST (de Jong et al. 2012), Euclid (Laureijs et al. 2011), and the SDSS IV 'Black Hole Mapper' program (Kollmeier et al. 2017), which will focus also on the optical characterization of eROSITA X-ray sources. Performing a dynamical analysis on numerical simulations will enable significant improvements in the assessment of further systematic uncertainties, such as the impact of residual interlopers in our sample, departures from virial equilibrium, and variation of the velocity anisotropy profile (Capasso et al., in preparation).

\section{ACKNOWLEDGEMENTS}

The Munich group acknowledges the support by the Cluster of Excellence 'Origin and Structure of the Universe', the MPG faculty fellowship program, and the Ludwig-Maximilians-Universität Munich. RC acknowledges participation in the IMPRS on Astrophysics at the Ludwig-Maximilians University and the associated financial support from the Max-Planck Society. RC and VS acknowledge support from the German Space Agency (DLR) through Verbundforschung project ID 50OR1603. AS is supported by the ERC-StG 'ClustersXCosmo', grant agreement 716762, and by the FAREMIUR grant 'ClustersXEuclid' R165SBKTMA. RC acknowledge financial support from the ERC-StG 'ClustersXCosmo', grant agreement 716762. AB acknowledges the hospitality of the LMU and partial financial support from PRIN-INAF 2014 'Glittering kaleidoscopes in the sky: the multifaceted nature and role of Galaxy Clusters?', P.I.: Mario Nonino.

Funding for the Sloan Digital Sky Survey IV has been provided by the Alfred P. Sloan Foundation, the U.S. Department of Energy Office of Science, and the Participating Institutions. SDSS-IV acknowledges support and resources from the Center for HighPerformance Computing at the University of Utah. The SDSS web site is www.sdss.org.

SDSS-IV is managed by the Astrophysical Research Consortium for the Participating Institutions of the SDSS Collaboration including the Brazilian Participation Group, the Carnegie Institution for Science, Carnegie Mellon University, the Chilean Participation Group, the French Participation Group, Harvard-Smithsonian Center for Astrophysics, Instituto de Astrofísica de Canarias, The Johns Hopkins University, Kavli Institute for the Physics and Mathematics of the Universe (IPMU) / University of Tokyo, the Korean Participation Group, Lawrence Berkeley National Laboratory, Leibniz Institut für Astrophysik Potsdam (AIP), MaxPlanck-Institut für Astronomie (MPIA Heidelberg), Max-PlanckInstitut für Astrophysik (MPA Garching), Max-Planck-Institut für Extraterrestrische Physik (MPE), National Astronomical Observatories of China, New Mexico State University, New York University, University of Notre Dame, Observatário Nacional / MCTI, The
Ohio State University, Pennsylvania State University, Shanghai Astronomical Observatory, United Kingdom Participation Group, Universidad Nacional Autónoma de México, University of Arizona, University of Colorado Boulder, University of Oxford, University of Portsmouth, University of Utah, University of Virginia, University of Washington, University of Wisconsin, Vanderbilt University, and Yale University.

\section{REFERENCES}

Allen S. W., Evrard A. E., Mantz A. B., 2011, ARA\&A, 49, 409

Andreon S., Serra A. L., Moretti A., Trinchieri G., 2016, A\&A, 585, A147

Baxter E. J. et al., 2018, MNRAS, 476, 2674

Becker M. R., Kravtsov A. V., 2011, ApJ, 740, 25

Beers T. C., Flynn K., Gebhardt K., 1990, AJ, 100, 32

Binney J., Tremaine S., 1987, Galactic dynamics. Princeton Univ. Press, Princeton, NJ, p. 747

Biviano A., Girardi M., 2003, ApJ, 585, 205

Biviano A., Salucci P., 2006, A\&A, 452, 75

Biviano A. et al., 2013, A\&A, 558, A1

Biviano A., Popesso P., Dietrich J. P., Zhang Y.-Y., Erfanianfar G., Romaniello M., Sartoris B., 2017, A\&A, 602, A20

Blanton M. R. et al., 2017, AJ, 154, 28

Bocquet S. et al., 2015, ApJ, 799, 214

Bocquet S., Saro A., Dolag K., Mohr J. J., 2016, MNRAS, 456, 2361

Bocquet S. et al., 2019, ApJ, 878, 55

Bulbul E. et al., 2019, ApJ, 871, 50

Capasso R. et al., 2019a, MNRAS, 488, 481

Capasso R. et al., 2019b, MNRAS, 482, 1043

Carlberg R. G. et al., 1997, ApJ, 485, L13

Clerc N. et al., 2016, MNRAS, 463, 4490

Corless V. L., King L. J., 2009, MNRAS, 396, 315

Dawson K. S. et al., 2016, AJ, 151, 44

de Haan T. et al., 2016, ApJ, 832, 95

de Jong R. S. et al., 2012, in McLean I. S., Ramsay S. K., Takami H., eds, Proc. SPIE Conf. Ser. Vol. 8446, Ground-based and Airborne Instrumentation for Astronomy IV. SPIE, Bellingham, p. 84460T

Diemer B., 2018, ApJS, 239, 35

Dietrich J. P. et al., 2019, MNRAS, 483, 2871

Dutton A. A., Macciò A. V., 2014, MNRAS, 441, 3359

Eddington A. S., 1913, MNRAS, 73, 359

Farahi A. et al., 2018, A\&A, 620, A8

Feroz F., Hobson M. P., 2008, MNRAS, 384, 449

Feroz F., Hobson M. P., Bridges M., 2009, MNRAS, 398, 1601

Feroz F., Hobson M. P., Cameron E., Pettitt A. N., 2019, The Open Journal of Astrophysics, 2, 10

Finoguenov A. et al., 2019, preprint (arXiv:1912.03262)

Gao L., De Lucia G., White S. D. M., Jenkins A., 2004, MNRAS, 352, L1

Giles P. A. et al., 2016, A\&A, 592, A3

Giles P. A. et al., 2017, MNRAS, 465, 858

Haiman Z., Mohr J. J., Holder G. P., 2001, ApJ, 553, 545

Hasselfield M. et al., 2013, J. Cosmol. Astropart. Phys., 7, 008

Hennig C. et al., 2017, MNRAS, 467, 4015

Hoeting J. A., Madigan D., Raftery A. E., Volinsky C. T., 1999, Stat. Sci., 14,382

Katgert P., Biviano A., Mazure A., 2004, ApJ, 600, 657

Klein M. et al., 2018, MNRAS, 474, 3324

Klein M. et al., 2019, MNRAS, 488, 739

Kollmeier J. A. et al., 2017, preprint (arXiv:1711.03234)

Laureijs R. et al., 2011, preprint (arXiv:1110.3193)

Levi M. et al., 2013, preprint (arXiv:1308.0847)

Lieu M. et al., 2016, A\&A, 592, A4

Lima M., Hu W., 2005, Phys. Rev. D, 72, 043006

Malmquist K. G., 1920, Meddelanden fran Lunds Astron. Obs. Serie II, 22, 1

Mamon G. A., Łokas E. L., 2005, MNRAS, 363, 705 
Mamon G. A., Biviano A., Murante G., 2010, A\&A, 520, A30

Mamon G. A., Biviano A., Boué G., 2013, MNRAS, 429, 3079

Mantz A., Allen S. W., Ebeling H., Rapetti D., Drlica-Wagner A., 2010, MNRAS, 406, 1773

Mantz A. B. et al., 2015, MNRAS, 446, 2205

Mantz A. B. et al., 2016, MNRAS, 463, 3582

Mantz A. B., Allen S. W., Morris R. G., von der Linden A., 2018, MNRAS, 473, 3072

Maughan B. J., 2014, MNRAS, 437, 1171

McClintock T. et al., 2019, MNRAS, 482, 1352

Merloni A. et al., 2012, preprint (arXiv:1209.3114)

Merritt D., 1985, MNRAS, 214, 25P

Merritt D., 1987, ApJ, 313, 121

Mirkazemi M. et al., 2015, ApJ, 799, 60

Mortonson M. J., Hu W., Huterer D., 2011, Phys. Rev. D, 83, 023015

Munari E., Biviano A., Mamon G. A., 2014, A\&A, 566, A68

Nagarajan A. et al., 2019, MNRAS, 488, 1728

Navarro J. F., Frenk C. S., White S. D. M., 1996, ApJ, 462, 563

Osipkov L. P., 1979, Sov. Astron. Lett., 5, 42

Pacaud F. et al., 2007, MNRAS, 382, 1289

Planck Collaboration et al., 2014, A\&A, 571, A20

Popesso P. et al., 2015, A\&A, 574, A105

Powell, 2006, Large-Scale Nonlinear Optimization, Springer, US

Pratt G. W., Croston J. H., Arnaud M., Böhringer H., 2009, A\&A, 498, 361

Pratt G. W., Arnaud M., Biviano A., Eckert D., Ettori S., Nagai D., Okabe N., Reiprich T. H., 2019, Space Sci. Rev., 215, 25

Predehl P. et al., 2010, in Arnaud M., Murray S. S., Takahashi T., eds, Proc. SPIE, Vol. 7732, Space Telescopes and Instrumentation 2010: Ultraviolet to Gamma Ray. SPIE, Bellingham, p. 77320U

Rykoff E. S. et al., 2012, ApJ, 746, 178

Rykoff E. S. et al., 2014, ApJ, 785, 104

Saro A., Mohr J. J., Bazin G., Dolag K., 2013, ApJ, 772, 47

Springel V., White S. D. M., Tormen G., Kauffmann G., 2001, MNRAS, 328,726

Staniszewski Z. et al., 2009, ApJ, 701, 32

Tinker J., Kravtsov A. V., Klypin A., Abazajian K., Warren M., Yepes G., Gottlöber S., Holz D. E., 2008, ApJ, 688, 709

Tiret O., Combes F., Angus G. W., Famaey B., Zhao H. S., 2007, A\&A, 476, L1

Truong N. et al., 2018, MNRAS, 474, 4089

Umetsu K., Broadhurst T., Zitrin A., Medezinski E., Coe D., Postman M., 2011, ApJ, 738, 41

van der Marel R. P., Magorrian J., Carlberg R. G., Yee H. K. C., Ellingson E., 2000, AJ, 119, 2038

Vikhlinin A. et al., 2009, ApJ, 692, 1060

Vikhlinin A. et al., 2009, ApJ, 692, 1033

Voges W. et al., 1999, A\&A, 349, 389

White S. D. M., Efstathiou G., Frenk C. S., 1993, MNRAS, 262, 1023

\section{APPENDIX A: TEST OF FIXED $c_{\mathrm{GAL}}$}

In Section 3.6 we described how we account for the number density profile $v(r)$ of the tracer population. We assume that the number density profile of the red sequence population is described by an NFW model, with a concentration for cluster galaxies of $c_{\text {gal }}=$ $5.37_{-0.24}^{+0.27}$ Hennig et al. (2017). We test the impact of this assumption on our results, performing our analysis on a range of concentrations. In Fig. A1 we show that the constraints of the scaling relation parameters are not very sensitive to the choice of the concentration parameter. The grey dashed lines correspond to the results obtained by fixing $c_{\text {gal }}$ to the above mentioned value from Hennig et al. (2017).

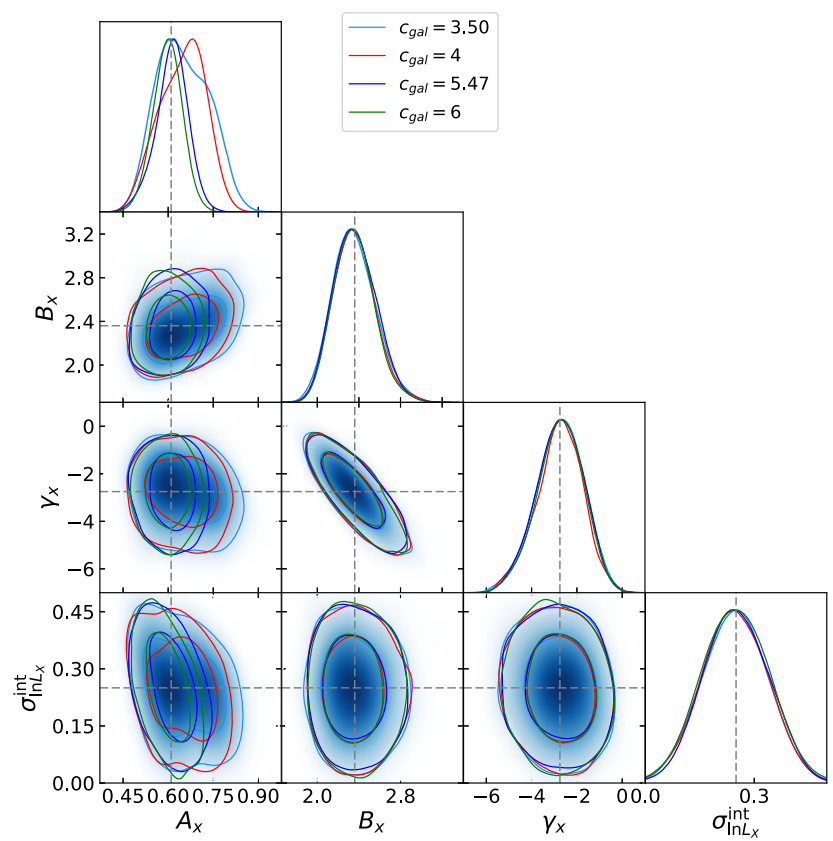

Figure A1. Posterior distribution of the scaling relation parameters for varying values of $c_{\text {gal }}$. Contours show the $1 \sigma, 2 \sigma$, and $3 \sigma$ confidence regions. The grey dashed lines correspond to the results obtained by fixing the concentration of cluster galaxies to $c_{\text {gal }}=5.37$.

This paper has been typeset from a $\mathrm{T}_{\mathrm{E}} \mathrm{X} / \mathrm{LT} \mathrm{E} \mathrm{X}$ file prepared by the author. 